\title{
Valoración del estado nutricional y consumo alimentario de los pacientes en terapia renal sustitutiva mediante hemodiálisis
}

\author{
$M^{a}$ Concepción Pereira Feijoo, Lidia Queija Martínez, Andrés Blanco Pérez, Ignacio Antonio Rivera Egusquiza, \\ Victoria Eugenia Martínez Maestro, Zaira Prada Monterrubio \\ Diplomadas/os de enfermería, Centros Hemodiálisis FRIAT Orense: Santa María, 0 Barco de Valdeorras, Verín/os \\ Salgueiros. España
}

\section{Resumen}

Introducción: La malnutrición proteico-energética es un hecho frecuente entre los pacientes en tratamiento sustitutivo renal con hemodiálisis y a ello contribuye, además de la propia técnica dialítica, la dieta inadecuada.

Objetivos: Valorar la evolución del estado nutricional y la ingesta alimentaria de los pacientes de 3 unidades de hemodiálisis extrahospitalaria, tras una intervención enfermera educativa.

Pacientes y métodos: Hemos realizado un estudio transversal en 66 pacientes de 3 unidades extrahospitalarias (44 hombres y 23 mujeres) con dosis de diálisis medida por KT/V > 1,3. Se hizo una determinación del estado nutricional por métodos convencionales y del consumo alimentario mediante encuesta dietética, determinándose mediante soporte informático el índice de alimentación saludable, identificando los déficits y/o excesos de nutrientes en cada paciente.

Se llevaron a cabo intervenciones educativas personalizadas dirigidas a corregir los excesos o déficits de nutrientes detectados en los pacientes, y se volvieron a hacer las mismas valoraciones a los seis meses.

Resultados: La prevalencia de desnutrición es leve en el $41 \%$ de hombres y $43 \%$ de las mujeres y moderada en el $34 \%$ de los hombres y $21 \%$ de las mujeres. El índice de alimentación saludable era inadecuado en el $37,31 \%$ de los pacientes en el análisis basal y postintervención educativa paso al $18,8 \%$.

\footnotetext{
Correspondencia:

$\mathrm{M}^{\mathrm{a}}$ Concepción Pereira Feijoo

Avda. de Portugal $n^{\circ} 100,5^{\circ} \mathrm{B}$

32002 Orense

E-mail: feijoopereira@gmail.com
}

La ingesta media energética es elevada (1398.86 $\mathrm{Kcal} /$ paciente/día) con alto consumo de colesterol y déficit de aminoácidos. Se detectó un alto consumo de hidratos de carbono simples ( $>20 \%$ ) y un elevado uso de proteínas de origen animal. El contenido mineral y vitamínico de la dieta es muy deficiente, destacando el bajo consumo de Vitamina $D(1.45 \pm 2.55 \mathrm{ng})$ y elevado de fósforo $(1052.28 \pm 356.23 \mathrm{~m} / \mathrm{día})$.

Conclusion: Tras una intervención educativa sobre nuestros pacientes, corrigiendo hábitos dietéticos no saludables, hemos conseguido mejorar su estado nutricional, reduciendo la prevalencia de desnutrición en nuestras unidades.

PALABRAS CLAVE

- VALORACIÓN NUTRICIONAL

- HEMODIÁLISIS

- ENCUESTA ALIMENTARIA

Assessment of nutritional status and food intake of patients in renal replacement therapy with hemodialysis

\section{Abstract}

Introduction: Protein-energy malnutrition is a common occurrence among patients on renal replacement therapy with hemodialysis and this in addition to the dialysis technique itself, improper diet contributes.

Objective: Assess the evolution of nutritional status and food intake of patients from 3 outpatient hemodialysis units, after an educational nurse intervention.

Methods: We conducted a cross-sectional study in 66 outpatient units from 3 patients (44 men and 23 
women) with dialysis dose measured by $\mathrm{Kt} / \mathrm{V}>1.3$. It was made a determination of nutritional status by conventional methods and food consumption by a diet questionnaire, determining by computer support the healthy eating index, identifying deficits and / or excess nutrients in each patient.

Customized educational interventions designed to correct the excesses or deficiencies of nutrients detected in patients were carried out. The same assessments were made at six months.

Results: The prevalence of malnutrition was mild in $41 \%$ of men and $43 \%$ of women and moderate in $34 \%$ of men and $21 \%$ of women. The healthy eating index was inadequate in $37.31 \%$ of patients at baseline but after the educational intervention was $18.8 \%$.

The average energy intake was high (1398.86 Kcal / patient / day) with high intake of cholesterol and amino acid deficiency. A high intake of simple carbohydrates ( $>20 \%$ ) and a high use of animal protein were detected. The mineral and vitamin content of the diet was very poor, highlighting the low consumption of vitamin D $(1.45 \pm 2.55 \mathrm{ng})$ and high phosphorus (1052.28 \pm $356.23 \mathrm{~m} /$ day).

Conclusion: After an educational intervention on our patients, correcting unhealthy dietary habits, we managed to improve their nutritional status, reducing the prevalence of malnutrition in our units.

\section{KEYWORDS}

\section{- NUTRITIONAL ASSESSMENT \\ - HEMODIALYSIS \\ - NUTRITION QUESTIONNAIRE}

\section{Introducción}

La enfermedad renal Crónica (ERC) es un síndrome con manifestaciones clínicas muy variadas que afecta a la mayor parte de los órganos y sistemas, lo cual refleja la complejidad de las funciones que el riñón desempeña en condiciones fisiológicas, así como de las severas consecuencias que comporta la disfunción renal. La insuficiencia renal, por tanto afecta a la situación metabólica nutricional de los pacientes ${ }^{1,2}$.

El desarrollo de las técnicas de diálisis ha supuesto un aumento en la supervivencia de estos pacientes, con una clara mejora de la calidad de vida, sin embargo estos avances hacen que los requerimientos nutriciona- les sean específicos y diferentes según la terapia renal sustitutiva (TRS) ${ }^{3}$.

La desnutrición proteico-energética es un problema clínico frecuente (20-25\%) en los pacientes con ERC sometidos a TRS mediante hemodiálisis (HD) ${ }^{4}$ y entre los factores condicionantes está la alimentación insuficiente $^{5}$. Es además uno de los mayores predictores de morbimortalidad de pacientes en HD, puesto que en los pacientes desnutridos, la morbimortalidad aumenta ${ }^{6}$.

Además de las condiciones de malnutrición debida a la ERC, la TRS contribuye de forma manifiesta por el stress catabólico, y en consecuencia el paciente en HD precisa unos requerimientos energéticos superiores a los sujetos normales, y a los urémicos no dializados $^{7,8,9}$.

Por tanto, la valoración del estado nutricional (EN) de estos pacientes debería ser incluida en la práctica habitual de control y seguimiento de los mismos. La monitorización clínica del EN y de la ingesta de nutrientes es fundamental en nuestros enfermos.

El estudio del consumo alimentario de estos pacientes es una herramienta básica que nos indica cómo el paciente cubre sus necesidades proteico-calóricas diarias $^{10}$.

El proceso de selección de los alimentos depende de la disponibilidad y aprendizaje, que va a permitir componer el menú diario según los gustos y hábitos del paciente. La alimentación es un proceso voluntario, educable, influido por factores socioeconómicos, psicológicos y geográficos., pero debe ser suficiente (cubrir todas las necesidades corporales), completa (contener todo tipo de nutrientes) y adecuada (adaptarse a cada edad, necesidad y consideraciones específicas de la enfermedad) ${ }^{11}$.

Por tanto, nos planteamos como objetivo determinar el EN de nuestros pacientes valorando su evolución, y la de la ingesta alimentaria que practican, tras una intervención enfermera educativa dirigida a corregir hábitos no saludables. 


\section{Material y métodos}

Se estudiaron los pacientes de tres unidades de HD con una permanencia mínima de seis meses en programa, desde septiembre de 2013 a abril de 2014 .

Se excluyeron los pacientes con procesos agudos, pacientes con problemas de compensación por déficit cognitivo, pacientes con ingresos hospitalarios durante el periodo de estudio, los transferidos a otras técnicas de depuración renal, y los pacientes que no aceptaron participar voluntariamente en el estudio.

Para la determinación del EN se utilizó la escala de Ulibarri (Tabla-1):

Tabla 1. Escala de Ulibarri ${ }^{(10)}$.

\begin{tabular}{|c|c|c|c|c|}
\hline \multirow{2}{*}{\multicolumn{5}{|c|}{$\begin{array}{l}\text { Valoración del grado de desnutrición (riesgo por desnutrición) } \\
\text { GRADO DE DESNUTRICIÓN }\end{array}$}} \\
\hline & & & & \\
\hline PARÁMETRO & NORMAL & LEVE & MODERADA & SEVERA \\
\hline $\begin{array}{l}\text { ALBÚMINA } \\
\text { SÉRICA(g/dl) } \\
\text { SCORE }\end{array}$ & $\begin{array}{c}3.5-4.5 \\
0\end{array}$ & $\begin{array}{c}3-3,49 \\
2\end{array}$ & $\begin{array}{c}2,5-2,9 \\
4\end{array}$ & $\begin{array}{c}<2,5 \\
6\end{array}$ \\
\hline $\begin{array}{l}\text { Linfocitos } \\
\text { totales/ml } \\
\text { SCORE }\end{array}$ & $\begin{array}{c}>1.600 \\
0\end{array}$ & $\begin{array}{c}1.200-1.599 \\
1\end{array}$ & $\begin{array}{c}800-1.200 \\
2\end{array}$ & $\begin{array}{c}<800 \\
3\end{array}$ \\
\hline $\begin{array}{l}\text { Colesterol } \\
\text { (mg/dl) } \\
\text { SCORE }\end{array}$ & $\begin{array}{c}>180 \\
0\end{array}$ & $\begin{array}{l}140-180 \\
1\end{array}$ & $\begin{array}{c}100-139 \\
2\end{array}$ & $\begin{array}{c}<100 \\
3\end{array}$ \\
\hline \multirow[t]{3}{*}{$\begin{array}{l}\text { Puntuación } \\
\text { total del filtro }\end{array}$} & $0-1$ & $2-4$ & $5-8$ & $<8$ \\
\hline & \multicolumn{4}{|c|}{ INTERPRETACIÓN } \\
\hline & $\begin{array}{l}\text { ALERTA } \\
\text { DE } \\
\text { DESNUTRI- } \\
\text { CIÓN }\end{array}$ & $\begin{array}{c}0-4 \\
\text { ALERTA } \\
\text { BAJA }\end{array}$ & $\begin{array}{l}5-8 \\
\text { ALERTA } \\
\text { MODERADA }\end{array}$ & $\begin{array}{l}\text { 9-12 } \\
\text { ALERTA } \\
\text { ALTA }\end{array}$ \\
\hline
\end{tabular}

Para evaluar el consumo alimentario de nuestros pacientes se utilizó un Diario Dietético de 3 días, autoadministrado. Los pacientes se adiestraron para anotar todos los alimentos ingeridos (sólidos y líquidos) pesados, y anotaron el tipo de alimento y forma de cocinado. El registro se hizo inmediatamente después de comer sin olvidar tentempiés, picoteos, aliños etc.

Una vez entregadas se revisaron la totalidad de las encuestas alimentarias comprobando la correcta recogida de información, ayudando al paciente a terminar de cumplimentarla resolviendo las posibles dudas que pudieran generar una encuesta incompleta e incorrecta.
Las encuestas se volcaron a un programa informático denominado DIAL, para su análisis.

Con el programa informático DIAL, se transforman los datos recogidos con la encuesta en energía y nutrientes, y además define un índice de alimentación saludable, teniendo en cuenta los parámetros que se muestran en la tabla 2, y en los requerimientos nutricionales de los pacientes en HD, expresados en la tabla 3.

Tabla 2. Índice de alimentación saludable ${ }^{(2)}$.

\begin{tabular}{|l|l|l|}
\hline PUNTUACIÓN DE O A 100 & $\begin{array}{l}\text { PUNTUACIÓN } \\
\text { MÍNIMA (0) }\end{array}$ & $\begin{array}{l}\text { PUNTUACIÓN } \\
\text { MÁXIMA (10) } \\
\text { Cuando se toma } \\
\text { la cantidad aconsejada }\end{array}$ \\
\hline Consumo de cereales & 0 raciones/día & 6 -10 raciones/día \\
\hline Consumo de vegetales & 0 raciones/día & $3-5$ raciones/día \\
\hline Consumo de frutas & 0 raciones/día & $2-4$ raciones/ día \\
\hline Consumo de lácteos & 0 raciones/día & $2-3$ raciones/día \\
\hline Consumo de carnes & 0 raciones/día & $2-3$ raciones/día \\
\hline Grasa total & $>45 \%$ energía & $<=30$ energía \\
\hline Grasa saturada & $>15 \%$ energía & $<0$ Energía \\
\hline Colesterol & $>450$ mg/día & $<300$ mg/día \\
\hline Sodio & $>4800$ mg/día & $<2400$ mg/día \\
\hline Variedad & $<=6$ alimentos/3 días & $>=16$ alimentos/ 3 días \\
\hline PUNTUACIóN & $>80$ DIETA EXCELENTE \\
\hline & $71-80$ MUY BUENA \\
\hline & $61-70$ BUENA & \\
\hline & $51-60$ ACEPTABLE & \\
\hline & $0-50$ ADECUADA & \\
\hline
\end{tabular}


Tabla 3. Requerimientos nutricionales en hemodiálisis 3,4 y 5$)$.

\begin{tabular}{|c|c|}
\hline $\begin{array}{l}\text { ENERGÍA } \\
\text { Kcal/día }\end{array}$ & $\begin{array}{l}35 \\
\text { (tener en cuenta, edad, talla, peso } \\
\text { y actividad física) }\end{array}$ \\
\hline $\begin{array}{l}\text { PROTEÍNAS } \\
\text { (g/Kg/día) }\end{array}$ & $\begin{array}{l}1.2-1.4 \\
(>50 \% \text { de alto valor biológico) }\end{array}$ \\
\hline GRASAS & $\begin{array}{l}30 \% \text { del aporte calórico total } \\
\text { (Saturadas }<10 \% \text { ) }\end{array}$ \\
\hline $\begin{array}{l}\text { FIBRA } \\
\text { (g/día) }\end{array}$ & $20-25$ \\
\hline $\begin{array}{l}\text { FLUIDOS } \\
(\mathrm{ml})\end{array}$ & Balance hídrico + $500 \mathrm{ml}$ \\
\hline $\begin{array}{l}\text { IONES } \\
\text { (individualizado) }\end{array}$ & $\begin{array}{l}\text { Na: } 2-3 \text { gr/día } \\
\mathrm{K}: 40 \mathrm{mg} / \mathrm{Kg} / \mathrm{día}\end{array}$ \\
\hline MINERALES & $\begin{array}{l}\text { Ca: } 1000-1500 \mathrm{mg} / \mathrm{día} \\
\text { (individualizado en relación con cifras de fósforo, } \\
\text { paratirina y dosis de vitamina } \mathrm{D} \text { ) } \\
\mathrm{P}: 15 \mathrm{mg} / \mathrm{Kg} / \mathrm{día} \\
\text { Mg: } 200-300 \mathrm{mg} / \text { día }\end{array}$ \\
\hline VITAMINAS & $\begin{array}{l}\text { Requerimientos aumentados de vitaminas } \\
\text { hidrosolubles } \\
\text { VITAMINA C: } 50 \mathrm{mg} / \mathrm{día} \\
\text { ÁCIDO FÓLIC0: } 1 \mathrm{mg} / \mathrm{día} \\
\text { VITAMINA B6: } 10 \mathrm{mg} / \mathrm{día} \\
\text { VITAMINA B12: } 5 \mathrm{mg} / \mathrm{día} \\
\text { VITAMINA D: } 67,2 \mathrm{mg} / \mathrm{día}\end{array}$ \\
\hline
\end{tabular}

Los diarios dietéticos de 3 días se entregaron en dos fases:

$1^{a}$ : Inicio del estudio: entrega, valoración energéticacalórica con aplicación informática. Identificación de déficits, necesidades y malos hábitos de nuestros pacientes.

Una vez finalizado el análisis de las encuestas, establecimos un programa para reforzamiento positivo de los hábitos nutricionales correctos y educación sanitaria para el cambio de hábitos nutricionales incorrectos, en cada uno de los pacientes incluidos en el estudio.

$2^{a}$ : A los 6 meses de la primera fase, y tras la intervención con educación sanitaria.

Además de los datos nutricionales, se recogieron datos demográficos, bioquímicos y KT/V registrados en la historia clínica del paciente, utilizando el programa informático de gestión de historias $\mathrm{Nefrosoft}^{\oplus}$.

También registramos la comorbilidad de cada paciente, calculada con el índice de Charlson 6 , y el índice tobillo- brazo derecho e izquierdo medido con un Doppler portátil con frecuencia de emisión de entre 8 y $10 \mathrm{Mhz}$.

En el análisis estadístico de los datos las variables cuantitativas se expresan como media +- DS y las variables cualitativas se expresan como frecuencia absoluta y frecuencia relativa.

Se realizó la prueba $t$ de student para muestras apareadas para comprobar si hay diferencias en los niveles medios de energía y nutrientes (antes y después de la intervención).

Se realizó el test de Mc-Nemar para comprobar si hay diferencias en los índices de alimentación saludable (antes y después de la intervención).

En todos los análisis se consideraron estadísticamente significativas las diferencias con $p<0.05$.

Los análisis se realizaron utilizando el software libre $R$ (http://www.r-project.org).

\section{Resultados}

De los 79 pacientes estudiados, 66 cumplían los criterios de inclusión establecidos.

Las causas de exclusión fueron fallecimiento $(n=4)$, trasplante $(n=1)$, transferencia a diálisis peritoneal $(n=1)$, transferencia a hemodiálisis diaria domiciliaria $(n=1)$, proceso intercurrente agudo $(n=5)$, no voluntariedad $(n=1)$.

44 eran hombres (65.67\%) y 23 mujeres $(34.33 \%)$ con una media de edad de $69.16(38 \pm 84)$.

El tiempo de permanencia en hemodiálisis era de 54.79 meses al inicio del estudio.

Las etiologías de fallo renal es la siguiente: 8 pacientes nefropatía glomerular (12.14\%), 5 pacientes nefropatía Intersticial $(7.59 \%), 5$ pacientes EPQ $(7.59 \%), 11$ nefropatía isquémica (16.7\%), 18 pacientes con nefropatía diabética $(27.29 \%)$ y 17 pacientes $(28.69 \%)$ con otro tipo de patología base (formas congénitas no incluidas en apartados anteriores y etiología no filiada).

La media de Kt/V de los pacientes a estudio fue de 1.8.

EI IMC medio, calculado con el programa informático DIAL, fue de 27.3 sin diferencias significativas entre 
sexos. Un 39\% de los pacientes tenían un peso normal (IMC 18.5-24.9), un 45\% con sobrepeso (IMC 2529.9 ), un $15 \%$ de obesos (IMC $>30$ ) y un $1 \%$ con peso insuficiente (IMC $<18.5$ ), siendo significativo el porcentaje de pacientes con sobrepeso.

Referente al índice de comorbilidad de Charlson el promedio es de 6.1 ( $D S \pm 1.65$ ) lo que indica que la mayoría de nuestros pacientes presentan alta comorbilidad.
El promedio de ITB/derecho es de 1.031 y el promedio de ITB/izquierdo es de 1.126, dentro de la normalidad (0.9-1.24), no encontrando por tanto relación con los patrones nutricionales.

EI EN medido por índice de Ulibarri se muestran en la Tabla-4:

Tabla 4. EN según Índice de Ulibarri.

\begin{tabular}{|c|c|c|c|c|}
\hline PACIENTES & NORMAL & DESNUTRICIÓN LEVE & DESNUTRICIÓN MODERADA & DESNUTRICIÓN SEVERA \\
\hline HOMBRES $65.67 \%$ & $20.6 \%$ & $41 \%$ & $37.4 \%$ & $1 \%$ \\
\hline MUJERES 34.33\% & $33.7 \%$ & $43 \%$ & $23.3 \%$ & $0 \%$ \\
\hline
\end{tabular}

Se observa un porcentaje alto de desnutrición en grado leve-moderado de los pacientes en estudio, siendo la desnutrición moderada, más frecuente en hombres, y en la desnutrición leve no hay diferencias significativas.
Se valoró también la albúmina en solitario (Tabla- 5), como valoración de la nutrición proteica (en el índice anterior forma parte de la valoración junto con otros componentes):

Tabla 5. EN y tasa de Albúmina Plasmática.

\begin{tabular}{|c|c|c|c|c|}
\hline PACIENTES & NORMAL & $\begin{array}{c}\text { DESNUTRICIÓN LEVE } \\
2.8-3.5 \mathrm{~g} / \mathrm{dl}\end{array}$ & $\begin{array}{c}\text { DESNUTRICIÓN MODERADA } \\
2.1-2.7 \mathrm{gr} / \mathrm{dl}\end{array}$ & $\begin{array}{c}\text { DESNUTRICIÓN SEVERA } \\
>2.1 \mathrm{gr} / \mathrm{dl}\end{array}$ \\
\hline HOMBRES 65.67\% & $22.8 \%$ & $36.6 \%$ & $40.6 \%$ & $0 \%$ \\
\hline MUJERES 34.33\% & $21.6 \%$ & $39.7 \%$ & $38.7 \%$ & $0 \%$ \\
\hline
\end{tabular}

En esta valoración, se constata un alto porcentaje de desnutrición proteica leve y moderada, sin diferencias significativas entre sexos. La nutrición proteica por tanto es deficiente, condicionada a su vez también como la ingesta suficiente de energía.

El Índice de alimentación saludable de los pacientes estudiados en la primera fase: 16 (23.88\%) tienen un índice de alimentación aceptable, 17 buena (25.37\%) ,2 excelente $(2.99 \%), 7$ muy buena $(10.45 \%)$ y 25 inadecuada $(37.31 \%)$.

En la segunda fase, con la intervención de educación sanitaria nutricional por parte del equipo sanitario del centro, se obtienen los siguientes resultados: 27 aceptable $(40.91 \%), 15$ buena $(22.73 \%), 5$ excelente $(7.58 \%), 7$ muy buena (10.61\%) y 12 inadecuada (18.18\%), disminuyendo en un porcentaje significativo los pacientes que tenían una dieta inadecuada.
En la Tabla-6, se muestran los datos comparativos de los diarios alimentarios referentes a la ingesta de energía y distintos nutrientes expresados como media y desviación típica por persona y día.

La ingesta media energética es de $1398.86 \mathrm{Kcal} / \mathrm{pa}-$ ciente/día El porcentaje diferencial entre el aporte y la ingesta recomendada es alto, se concreta en un rango de 60 y $65 \%$, porcentaje importante dado que los pacientes objeto de estudio no tenían ningún proceso intercurrente agudo. 
Tabla 6. Ingesta media diaria de energía y nutritientes.

\begin{tabular}{|c|c|c|c|}
\hline & BASAL & POST & P-valor \\
\hline Nutrientes & $(n=66)$ & $(n=66)$ & \\
\hline Energía (Kcal) & $1398.86 \pm 645.42$ & $1471.30 \pm 397.62$ & 0.4318 \\
\hline IR & $2354.76 \pm 405.94$ & $2324.95 \pm 400.32$ & 0.6668 \\
\hline$\%$ AP/IR & $59.52 \pm 25.98$ & $64.93 \pm 21.21$ & 0.1989 \\
\hline Proteína (g) & $90.99 \pm 137.51$ & $95.87 \pm 172.77$ & 0.8596 \\
\hline $\mathrm{H}$ de $\mathrm{C}(\mathrm{g})$ & $118.46 \pm 88.37$ & $132.25 \pm 51$ & 0.2702 \\
\hline Fibra (g) & $16.11 \pm 14.99$ & $14.92 \pm 6.63$ & 0.5697 \\
\hline Grasa T (g) & $65.56 \pm 34.14$ & $72.56 \pm 66.15$ & 0.4666 \\
\hline AGS (g) & $22.24 \pm 12.47$ & $22.54 \pm 8.08$ & 0.8739 \\
\hline AGM (g) & $30.57 \pm 16.98$ & $28.67 \pm 9.46$ & 0.4361 \\
\hline $\operatorname{AGP}(p)$ & $7.00 \pm 3.61$ & $7.62 \pm 2.53$ & 0.2613 \\
\hline AGP/AGS & $0.33 \pm 0.12$ & $0.36 \pm 0.11$ & 0.1981 \\
\hline$A G P+A G M / A G S$ & $1.77 \pm 0.54$ & $1.71 \pm 0.44$ & 0.5093 \\
\hline CT (mg) & $246.17 \pm 99.66$ & $292.77 \pm 100.40$ & $<0.05$ \\
\hline Agua (g) & $931.03 \pm 622.50$ & $909.38 \pm 275.66$ & 0.8018 \\
\hline Alcohol (g) & $1.28 \pm 4.49$ & $0.49 \pm 1.34$ & 0.1751 \\
\hline $\mathrm{Ca}(\mathrm{mg})$ & $631.98 \pm 255.51$ & $677.67 \pm 240.16$ & 0.3001 \\
\hline $\mathrm{Fe}(\mathrm{mg})$ & $10.53 \pm 5.91$ & $10.24 \pm 3.75$ & 0.7565 \\
\hline Yodo (ng) & $71.89 \pm 27.33$ & $83.18 \pm 34.39$ & $<0.05$ \\
\hline Magnesio(mg) & $188.86 \pm 78.13$ & $206.03 \pm 53.40$ & 0.1334 \\
\hline Zinc(mg) & $7.47 \pm 3.35$ & $8.09 \pm 2.72$ & 0.2585 \\
\hline Selenio(ng) & $79.26 \pm 37.6$ & $112.66 \pm 177.31$ & 0.1487 \\
\hline $\mathrm{Na}(\mathrm{mg})$ & $1780.89 \pm 1059.18$ & $2006.54 \pm 927.02$ & 0.1441 \\
\hline $\mathrm{K}(\mathrm{mg})$ & $2214.92 \pm 1059.18$ & $2264.06 \pm 711.13$ & 0.734 \\
\hline$P(m g)$ & $1052.28 \pm 356.23$ & $1148.22 \pm 327.39$ & 0.1056 \\
\hline Flúor(mg) & $190.02 \pm 96.75$ & $202.033 \pm 70.68$ & 0.4174 \\
\hline Vitamina $\mathrm{Bl}(\mathrm{mg})$ & $1.05 \pm 0.63$ & $1.022 \pm 0.36$ & 0.6804 \\
\hline Vitamina B2(mg) & $1.96 \pm 0.57$ & $1.38 \pm 0.55$ & 0.634 \\
\hline Niacina (mg) & $26.71 \pm 10.81$ & $30.48 \pm 9.00$ & $<0.05$ \\
\hline Vitamina B6(mg) & $13.70 \pm 94.38$ & $2.33 \pm 6.47$ & 0.1656 \\
\hline Ácido Fólico (ng) & $206.08 \pm 164.48$ & $201.61 \pm 96.33$ & 0.8263 \\
\hline Vitamina Bl2(ng) & $5.76 \pm 15.73$ & $5.52 \pm 9.70$ & 0.2235 \\
\hline Vitamina C (mg) & $74.09 \pm 80.76$ & $66.62 \pm 37.37$ & 0.8425 \\
\hline Retinol(ng) & $380.36 \pm 1428.40$ & $560.35 \pm 2028.20$ & 0.5648 \\
\hline Carotenos (ng) & $2479.7 \pm 2394.41$ & $2809.35 \pm 2095.92$ & 0.4217 \\
\hline Vitamina A(ng) & $720.96 \pm 525.91$ & $897.06 \pm 1551.07$ & 0.8618 \\
\hline Ác. Pantoteico(mg) & $3.91 \pm 1.64$ & $4.41 \pm 1.57$ & 0.0734 \\
\hline Biotina(ng) & $17.67 \pm 12.02$ & $20.73 \pm 11.35$ & 0.1309 \\
\hline Vitamina D (ng) & $1.45 \pm 2.55$ & $1.43 \pm 2.59$ & 0.844 \\
\hline Vitamina E (mg) & $4.54 \pm 2.78$ & $4.57 \pm 1.98$ & 0.3751 \\
\hline
\end{tabular}

IR: Ingesta diaria recomendada.

\%AP/IR: Porcentaje del aporte actual respecto de la ingesta recomendada.
La distribución del aporte energético es:

El consumo de grasas está por encima del rango recomendado para nuestros pacientes ( $>$ del $30 \%$ ). El dato del colesterol total es Ilamativo, muy por encima de la recomendación de $100-120 \mathrm{mg} / \mathrm{Kcal}$, el nivel medio de colesterol cuantificado en base a la dieta de los pacientes es alto, más incluso en la segunda intervención, teniendo en cuenta además que un $52 \%$ de los mismos están con tratamiento farmacológico para mantener niveles adecuados de este parámetro.

La ingesta observada de ácidos grasos monoinsaturados es la más elevada de las distintas familias de ácidos grasos. Los diarios alimenticios nos revelan al aceite de oliva como principal grasa de adición, constituyendo la principal fuente de ácidos grasos monoinsaturados.

En cuanto a los hidratos de carbono destacar que alrededor de un $20 \%$ de la ingesta de estos procede de hidratos de carbono simples ( $\mathrm{La}$ recomendación es de no sobrepasar un 10\%).

El consumo de proteínas, como comentamos anteriormente está bastante por encima de los requisitos estimados, los diarios alimenticios nos indican que en torno a un $80 \%$ es de origen animal (31\% carne, $27 \%$ pescados, $11 \%$ huevos y un $10 \%$ lácteos).

En relación al contenido mineral, el consumo de calcio está disminuido, y el del potasio $(2214,92+-1059,18 \mathrm{mg})$ y fósforo $(1052.28 \pm 356.23 \mathrm{mg})$, es muy elevado con respecto a las ingestas recomendadas para nuestros pacientes.

Respecto a las vitaminas, en general están disminuidas destacando esencialmente el déficit de Vitamina $D(1.45 \pm 2.55 \mathrm{ng} /$ día $)$ en cambio la vitamina $C$ está por encima de los $60 \mathrm{mg} /$ día recomendados con la posible acumulación de oxalatos asociada.

La ingesta de agua está sobrepasada ampliamente a lo recomendado, y el aporte de fibra es insuficiente.

También se observa que los principales grupos de alimentos que contribuyen al aporte energético son los cárnicos y derivados. 


\section{Discusión}

La prevalencia de desnutrición proteico-energética es frecuente en pacientes en programa de hemodiálisis, aunque es difícil de precisar a pesar de los diferentes métodos para su valoración ${ }^{12}$.

Nuestros resultados evidencian una insuficiente ingesta de energía acompañada de una ingesta excesiva de grasas, colesterol, potasio, fósforo y azúcares simples con una insuficiente ingesta de hidratos de carbono complejos, calcio y fibra.

La ingesta calórica en los pacientes en TRS, esencial para la síntesis proteica, al ser deficiente se destina a fines energéticos ${ }^{7,13}$.

Por tanto, el déficit crónico energético condiciona en el desarrollo de malnutrición, que en nuestros pacientes está presente en el $37,4 \%$ de los hombres y el $23,3 \%$ de las mujeres.

En nuestro trabajo como en la bibliografía existente sobre el tema, queda de manifiesto la importancia de conocer el estado nutricional de los pacientes en HD, como marcador de disminución de la calidad de vida y por el riesgo de morbimortalidad asociada, así como la importancia del papel de la enfermería en relación a la educación sanitaria de nuestros pacientes respecto a la ingesta adecuada de nutrientes, pues a pesar de los múltiples estudios que desvelan los déficit nutricionales, los consejos dietéticos continúan en la mayoría de los casos limitándose a restricciones, de ahí la importancia de conocer qué comen nuestros pacientes, para que el personal de enfermería pueda establecer unas recomendaciones nutricionales para cada uno de nuestros pacientes, estimulando una adecuada adherencia a la dieta ${ }^{3,14}$.

En nuestro estudio hay que destacar por llamativo que la ingesta energética se hace a expensas de la ingesta de grasas y es especialmente llamativa la ingesta de Colesterol: $246.17 \pm 99.66 \mathrm{mg} / \mathrm{dí}$ muy por encima de lo recomendado (100-200 mg/1000 Kcal).

También destaca la baja ingesta de fibra $(16.11 \pm 14.99$ g) elemento protector del Ca de colon.

El análisis del consumo alimentario nos muestra las deficiencias nutricionales de nuestros pacientes destacando en el capítulo de vitaminas en tremendo déficit de ácido Fólico (0,00026 mg/día ) frente a los requerimientos mínimos día de $1 \mathrm{mg} / \mathrm{día}$, y también en relación a Vitamina D, la ingesta es mínima $(0,0000145 \mathrm{mg} /$ día) lo cual explica el déficit crónico de vitamina $D$ nutricional de estos pacientes y las graves consecuencias para el metabolismo mineral que todavía se agrava más por la elevada ingesta de Fosforo (1052.28 \pm 356.23 mg/día) y baja de calcio ( $631.98 \pm 255.51 \mathrm{mg} / \mathrm{día})$.

Nuestra intervención dietética educativa, disminuyo significativamente el porcentaje de los pacientes que tenían una dieta inadecuada: $37.31 \%$ vs $18,18 \%$.

En relación al conocimiento y cumplimiento del tratamiento dietético, la percepción de los pacientes es elevada: un $91 \%$ dicen conocerlo y un $79 \%$ cumplirlo, los resultados obtenidos ponen de manifiesto que la realidad no es la misma. Un $69 \%$ de los pacientes encuestados no creen que la alimentación sea un factor importante para mejorar su salud, lo que pone de manifiesto que los pacientes de nuestras unidades desconocen la importancia que tiene la alimentación en el tratamiento de su enfermedad, debido principalmente a la falta de información, valorando más otros aspectos de su tratamiento como el medicamentoso.

El primer análisis de las encuestas pone de manifiesto también que el $36 \%$ de nuestros pacientes no hacen una de las comidas principales a causa de los horarios de su tratamiento y al decaimiento/fatiga posthemodiálisis.

Tras el abordaje de este tema por parte del personal de enfermería aconsejando disminuir el volumen y aumentar la frecuencia de las comidas, junto con la reorganización de los horarios de las comidas los días de hemodiálisis, en la segunda fase del estudio se rebajó dicho porcentaje al $15 \%$.

A la vista de esta situación, pensamos que desde el inicio de la terapia renal de hemodiálisis, debe hacerse una valoración nutricional para evitar llegar a situaciones avanzadas de desnutrición en las que cualquier intervención pierde eficacia.

La prevención de la malnutrición es de capital importancia, y enfermería juega un papel fundamental para ello, ofreciendo adecuada formación, información y control de las necesidades alimentarias del paciente.

El papel de la enfermera es fundamental en este sentido pero la cronicidad de la enfermedad y la dificultad que representa conseguir cambios en los hábitos dietéticos, pueden hacernos disminuir la atención en este tipo de aspectos, siendo fundamental no bajar la guardia puesto que una adecuada nutrición de los pacientes favorece una mejor calidad de vida, mejora la 
tolerancia al tratamiento y disminuye la comorbilidad asociada ${ }^{15}$.

Es mucho el tiempo en que la enfermera pasa con el paciente durante la realización de la técnica, y esto supone un mayor número de oportunidades para reforzar, cambiar o mejorar aspectos relacionados con la nutrición del paciente.

Nuestra experiencia nos hace ver que una adecuada intervención educativa es directamente proporcional a la eficacia y resultados en cuanto a aprendizaje nutricional del paciente se refiere.

El tiempo que los pacientes permanecen en contacto con nosotros durante las sesiones de diálisis, pensamos se debería optimizar poniendo en marcha estrategias educativas, estableciendo vínculos con los pacientes y familia, y propiciar la educación continua que se necesita para generar cambios en los hábitos del paciente a corto y largo plazo.

La malnutrición en HD es un problema a menudo subestimado, pues resulta difícil hacer un diagnóstico nutricional adecuado. Los métodos utilizados para dicha evaluación (IMC, determinaciones analíticas, encuestas terapéuticas...) no devuelven resultados categóricos pues están afectados por otros factores como enfermedades base, tratamientos y las variaciones de peso por la distribución de líquidos corporales que lleva implícita la hemodiálisis 5,10,12.

A la vista de nuestros resultados, y a pesar de las múltiples dificultades para la valoración nutricional de los pacientes en HD, podemos concluir que nuestro grupo ha puesto en marcha una metodología de trabajo en la que tras intervenciones educativas personalizadas sobre nuestros pacientes, corrigiendo hábitos dietéticos no saludables, hemos conseguido mejorar su estado nutricional, reduciendo la prevalencia de desnutrición en nuestras unidades.

Ya lo expresó Florence Nigthingale, en 1890: que para el cuidado de enfermos "Uso adecuado del calor, luz, limpieza.... Y la oportuna selección y administración de dietas".

\section{Agradecimientos}

A Alfonso Santiago Otero González, por su continuo apoyo y colaboración.
Recibido: 10 diciembre 2014

Revisado: 8 enero 2015

Modificado: 22 febrero 2015

Aceptado: 10 marzo 2015

\section{Bibliografía}

1. Stenvinkel P, Heimburger 0, Paultre F et al. Strong association between malnutrition, inflammation, and atherosclerosis in chronic renal failure. Kidney Int 1999; 55:1899-1911.

2. Suliman ME, Qureshi AR, Bárany $P$, Stenvinkel $P$, Filho JC, Anderstam B et al. Hiperhomocysteinemia, nutritional status, and cardiovascular disease in hemodiálisis patientes. Kidney Int 2000; 57:17271735.

3. K/DOQUI Clinical Practice Guidelines for nutrition in Chronic Renal Failure. Am J Kidney Dis.2000; 35 Suppl 2:s1-37.

4. M. J. Fernández (et al). Estado nutricional, comorbilidad e inflamación en hemodiálisis. Nefrología. Vol. XX. № 6. 2000.

5. Ortega Anta R, López Sobaler AM, Andrés Carvajales $\mathrm{P}$, Requejo Marcos, AM, Molinero Casares, LM. DIAL 1.0. Programa de evaluación de dietas y gestión de datos de alimentación. Alce Ingenieria, 2007, 107 pp.

6. Charlson ME, Pompei P, Ales KL, MacKenzie CR. A new method of classifying prognostic comorbidity in longitudinal studies: development and validation. $J$ Chronic Dis 1987; 40: 373-83.

7. Lorenzo V., Rufino M., Martín M. Aspectos nutricionales en hemodiálisis. En: Fernando Valderrábano. Tratado de hemodiálisis. Barcelona: MédicaJIMS; 1999; 339-360.

8. Marcen R, Martín del Río R, Teruel JL, Cosmes P. Quereda C, Ortuño J, Malnutrición Proteica pérdidas de aminoácidos durante la hemodiálisis Nefrología 5 (11) .51-55.1985.

9. Wiggins KL. Guidelines for Nutrition Care of Renal Patients. $3^{\text {a }}$.ed.Chicago: American Dietetic Association; 2002.

10. Ulíbarri JI, González-Madroño A, González A, Fernández G, Rodríguez F. Nuevo procedimiento para la detección precoz y control de la desnutrición hospitalaria. Nutr Hosp. 2002; 17:179-88. 
11. Gil, A.; Mañas, M., Martínez de Victoria, E. (2010). Ingestas dietéticas de referencia, objetivos nutricionales y guías. En libro: Tratado de nutrición. $2^{a}$ Ed. Tomo III Nutrición humana en el estado de salud. Madrid. Ed.Médica Panamericana SA, pp: 30-65.

12. J. M. Manzano (et al). Parámetros antropométricos más idóneos para valorar el Estado nutricional de los pacientes con insuficiencia renal crónica tratados con hemodiálisis en los centros periféricos. Rev. Soc. Enferm. Nefrol. 2003; (6) 3:6/15.

13. D. de Luis, J. Bustamante. Aspectos nutricionales en la Insuficiencia renal. Nefrología 2008; 28 (3)339-348.
14. Toigo G,AparicioM, Attman PO, Cano N, Cianciaruso $B$, Engel $B$, et al. Expert Working Group report on nutricion in adults patients with renal insufficiency (part 1 of 2). Clin Nutr.2000; 19: 197-207.

15. Documento SENPE-SEDOM sobre la codificación de la desnutrición hospitalaria. http://www.sedom. es/99_pdf/senpesedom.pdf.

16. Stevinkel $P$, Heimburger 0 , Lindholm B Kaysen GA, Bergström J. Are there two types of malnutrition in chronic renal failure? Nephrol Dial Transplant 2000; 15:953-960.

ANEXO. Diario dietético de 3 días.

\begin{tabular}{|c|c|c|c|c|}
\hline \multicolumn{2}{|l|}{ FECHA } & \multicolumn{3}{|l|}{ PACIENTE } \\
\hline \multicolumn{5}{|c|}{ PRIMER DÍA } \\
\hline DESAYUNO & ALIMENTO & PREPARACIÓN & MEDIDA CASERA & PESO APROXIMADO \\
\hline \multicolumn{5}{|c|}{ MEDIA MAÑANA } \\
\hline \multicolumn{5}{|c|}{ COMIDA } \\
\hline \multicolumn{5}{|l|}{ MERIENDA } \\
\hline \multicolumn{5}{|l|}{ CENA } \\
\hline \multicolumn{5}{|l|}{ NOCHE } \\
\hline \multicolumn{5}{|l|}{ ACEITE } \\
\hline \multicolumn{5}{|l|}{ PAN } \\
\hline \multicolumn{5}{|l|}{ EXTRAS } \\
\hline FECHA & PACIENTE & & & \\
\hline \multicolumn{5}{|c|}{ SEGUNDO DÍA } \\
\hline DESAYUNO & ALIMENTO & PREPARACIÓN & MEDIDA CASERA & PESO APROXIMADO \\
\hline \multicolumn{5}{|c|}{ MEDIA MAÑANA } \\
\hline \multicolumn{5}{|c|}{ COMIDA } \\
\hline \multicolumn{5}{|l|}{ MERIENDA } \\
\hline \multicolumn{5}{|l|}{ CENA } \\
\hline \multicolumn{5}{|l|}{ NOCHE } \\
\hline \multicolumn{5}{|l|}{ ACEITE } \\
\hline \multicolumn{5}{|l|}{ PAN } \\
\hline \multicolumn{5}{|l|}{ EXTRAS } \\
\hline FECHA & PACIENTE & & & \\
\hline \multicolumn{5}{|c|}{ TERCER DÍA } \\
\hline DESAYUNO & ALIMENTO & PREPARACIÓN & MEDIDA CASERA & PESO APROXIMADO \\
\hline \multicolumn{5}{|c|}{ MEDIA MAÑANA } \\
\hline \multicolumn{5}{|l|}{ COMIDA } \\
\hline \multicolumn{5}{|l|}{ MERIENDA } \\
\hline \multicolumn{5}{|l|}{ CENA } \\
\hline \multicolumn{5}{|l|}{ NOCHE } \\
\hline \multicolumn{5}{|l|}{ ACEITE } \\
\hline \multicolumn{5}{|l|}{ PAN } \\
\hline EXTRAS & & & & \\
\hline
\end{tabular}

